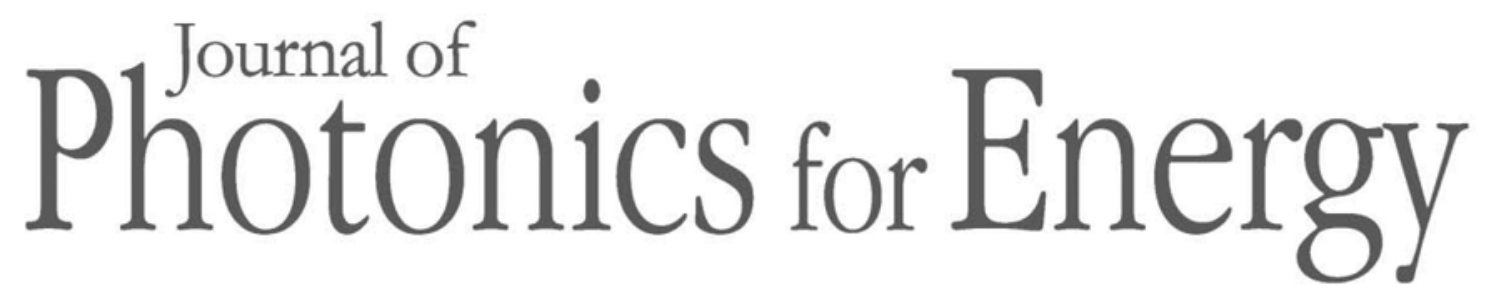

PhotonicsforEnergy.SPIEDigitalLibrary.org

\title{
JPE 2018 List of Reviewers
} SPE. $\begin{aligned} & \text { "JPE 2018 List of Reviewers," J. Photon. Energy 9(1), } 010101 \text { (2019), } \\ & \text { doi: 10.1117/1.JPE.9.010101. }\end{aligned}$ 
The Journal of Photonics for Energy would like to sincerely thank the following individuals who served as reviewers in 2018. The success of our publication hinges on the voluntary contributions of time and energy put forth by these professionals.

\begin{tabular}{|c|c|c|}
\hline Fahhad Alharbi & Ivan Kassal & Mikhail Raikh \\
\hline Arafa Aly & Yutaka Kawabe & R. Reisfeld \\
\hline John Ballato & Do Young Kim & Lewis Rothberg \\
\hline Supriyo Bandyopadhyay & Hannah Kim & Arvydas Ruseckas \\
\hline Shubhra Bansal & JongHyun Kim & Atsushi Sakurai \\
\hline Indrasen Bhattacharya & Achim Kittel & Marco Schiavon \\
\hline Wengang $\mathrm{Bi}$ & Rafael Kleiman & Timothy Schmidt \\
\hline Paul Blom & Anna Koehler & Anant Setlur \\
\hline Martin Bryce & Lisa Krayer & Chuanxiang Sheng \\
\hline Juan Casado & Evan Lafalce & Makoto Shimizu \\
\hline Jose Castillo Aguilella & Paul Lane & Mohamed Sid-Ahmed \\
\hline Song Chen & Guglielmo Lanzani & Ranjan Singh \\
\hline Xihan Chen & Frederic Laquai & James Sites \\
\hline Qiang Cheng & Nam Le & Mehmet Solmaz \\
\hline Cheng-Hsuan Chou & Gerald Ledru & Aloisi Somer \\
\hline Daniel Congreve & Tae-Woo Lee & Zhaoning Song \\
\hline Longji Cui & Tsung-Xian Lee & Shi-Jian Su \\
\hline Aleksandra Cvetkovic & Andrej Lenert & Yuehong Su \\
\hline Jeffrey D' Archangel & Gang Li & Jonathan Suen \\
\hline Enrico Da Como & Huamin $\mathrm{Li}$ & Oleg Sulima \\
\hline Xianming Dai & Jiayu Li & Dali Sun \\
\hline Niels Damrauer & Yao Li & Lech Sznitko \\
\hline Nathaniel Davis & Yongfang Li & Chunyan Tan \\
\hline Dick de Boer & Yuexiang Li & Yanpei Tian \\
\hline Barry Dunietz & David Lidzey & Jonathan Tong \\
\hline Vladimir Dyakonov & Hua Liu & Zeev Vardeny \\
\hline Guojia Fang & Xianglei Liu & Daniel Vazquez \\
\hline Maurizio Ferrari & Laurent Lombez & Victor Vedanayakam \\
\hline Daniel Feuermann & Sayani Majumdar & Robin Vismara \\
\hline Claudio Fontanesi & Masashi Mamada & Alexandre Walker \\
\hline Ben Fredj & Tom Markvart & Baomin Wang \\
\hline Jan Freudenberg & Jason McNeill & Liping Wang \\
\hline Daniel Friedman & Claiborne McPheeters & Zhijun Wang \\
\hline Moritz Futscher & Sean McSherry & Zhuoxian Wang \\
\hline Bhoj Gautam & Ajit K. Meikap & Shun Watanabe \\
\hline Shaolong Gong & Joseph Melinger & Franz-Peter Wenzl \\
\hline Nima Gorji & Takashi Minemoto & Keith Wilcox \\
\hline Zhe Guan & Shintaro Mizuno & Matthew Wilkins \\
\hline Tzung-Fang Guo & Ramesh Mohan & Markus Wohlgenannt \\
\hline Dongheon $\mathrm{Ha}$ & Margarita Mondragon & Kam Sing Wong \\
\hline Sidi Hamady & Ivan Moreno & Di Wu \\
\hline Kenneth Hanson & Nicola Morley & Donghai Wu \\
\hline Azwar Hayat & Dan Moses & Ting Wu \\
\hline Edwin Heilweil & Shuichi Nagamatu & Kaikai Xu \\
\hline Junming Ho & Tho Nguyen & Yunlu Xu \\
\hline Szuheng Ho & Christian Nielsen & Renqiang Yang \\
\hline Haifeng $\mathrm{Hu}$ & Deirdre O'Carroll & Zhenhai Yang \\
\hline Mohamad Hussin & Maria Belen Oviedo & Yuhan Yao \\
\hline Alan Huston & Shyam Pandey & Musa Yilmaz \\
\hline Uyen Huynh & Keunhan Park & Shuai Yong \\
\hline Hideo lizuka & Toni Pasanen & Haomiao Yu \\
\hline Kenji Ishizak & Akshit Peer & Zhi-Gang Yu \\
\hline Seigo Ito & Dmytro Perepichka & Anil Yuksel \\
\hline Klaus Jaeger & Igor Perepichka & Charlie Zhang \\
\hline Xiaomei Jiang & Shirish Pethe & TieJun Zhang \\
\hline Justin Johnson & William Potscavage & Zhuomin Zhang \\
\hline Gytis Juska & Qiquan Qiao & Bo Zhao \\
\hline Hemashilpa Kalagara & Kun Qin & Yitong Zhao \\
\hline Jason Karp & Yang Qin & Leize Zhu \\
\hline
\end{tabular}

